\title{
Seroprevalence of Novel Coronavirus SARS-CoV-2 at a Community Hospital Emergency Department and Outpatient Laboratory in Northern Orange County, California
}

\author{
Jason Yamaki ${ }^{1,2} \cdot$ Harry Peled $^{3} \cdot$ Sajen Mathews ${ }^{3} \cdot$ David Park $^{3} \cdot$ Mina Firoozi $^{3} \cdot$ Kim Smith $^{3} \cdot$ Lee Nguyen $^{3,4}$ (D
}

Received: 15 September 2020 / Revised: 28 October 2020 / Accepted: 28 October 2020 / Published online: 23 November 2020

(C) W. Montague Cobb-NMA Health Institute 2020

\begin{abstract}
Introduction The severe acute respiratory syndrome related coronavirus 2 (SARS-CoV-2) has infected more than 20 million people worldwide, and the spread is most prevalent in the USA, where California had accounted over 240,000 cases in the initial 5 months of the pandemic. To estimate the number of infected persons in our community, we conducted a cross-sectional study to estimate seroprevalence of SARS-CoV-2 infection.

Methods This cross-sectional study evaluated the presence of immunoglobulin G, antibody for SARS-CoV-2 during the time period of July 15, 2020, to July 27, 2020. Testing was done on serum samples from patients who had visited affiliated outpatient clinics or our emergency department. Additionally, we collected age, gender, ethnicity, race, and location of testing.

Results Eight hundred sixty-five tests were included in the study. The outpatient clinics cohort accounted for 56\% of results and emergency department (ED) contributed 44\%. The positive percentage of SARS-CoV-2 test was 9.4\% (95\% CI: 0.08-0.12). The positivity rates of the outpatient $(5.6 \%)$ and ED (14.2\%) setting differed. The prevalence of SARS-CoV-2 IgG was greatest in those that identified as Hispanic/Latino, $18.1 \%$ versus $13.4 \%$ in other groups. Specifically compared to the non-Hispanic/Latino population, the prevalence was significantly higher, with a relative risk of 2.73 (95\% CI: 1.8-4.1), $p<0.0001$.

Conclusion The low antibody positivity rate in the community indicates the need for a vaccine. The Hispanic/Latino patient population should be considered for increased education on preventing transmission and acquisition of COVID-19 as well as being considered as a priority for vaccination once a vaccine is available.
\end{abstract}

Keywords COVID-19 $\cdot$ SARS-CoV-2 · Immunoglobulin G

\section{Introduction}

The severe acute respiratory syndrome related coronavirus 2 (SARS-CoV-2) is the cause of the coronavirus disease 2019 (COVID-19) pandemic [1, 2]. The virus has caused more than 20 million infections worldwide, with global deaths nearing

Lee Nguyen

l.nguyen@uci.edu

1 Department of Pharmacy Practice, Chapman University School of Pharmacy, Irvine, CA, USA

2 Department of Pharmacy, Hoag Memorial Hospital Presbyterian, Newport Beach, CA, USA

3 Providence St. Joseph Health System, St. Jude Medical Center, Fullerton, CA, USA

4 Department of Clinical Pharmacy Practice, University of California, Irvine School of Pharmacy \& Pharmaceutical Sciences, CA Irvine, USA three-quarters of a million [3, 4]. Within the USA, the number of cases exceeds five million, and within California, cases exceeded 240,000 during the first 5 months of the pandemic [4]. The spread of COVID-19 infections has not distributed evenly throughout California, and the capacity for testing for COVID19 has limited an accurate assessment of prevalence in the community. Thus, the true prevalence in each community is uncertain, and there also exists a need for additional health literacy, understanding, and knowledge regarding the disease prevalence in each community to optimally develop targeted strategies [5]. To this, the Centers for Disease Control and Prevention (CDC) recommends understanding the characteristics of the community and its population, health system, and public health capacity in order to implement appropriate mitigation strategies specific to that community [6].

Based on the US Census Bureau, Orange County is considered a mildly diverse population with the majority of the population identifying as White non-Hispanic (71\%) or Asian non-Hispanic $(21.7 \%)$. However, the percent of the 
population that identifies as Hispanic/Latino in origin is $34 \%$ [7]. Previous reports have shown that racial minorities such as African American and Hispanics are disproportionately affected by COVID-19 as an association has been observed with these groups and acquisition of SARS-CoV-2 infection [8-10]. Data from the CDC Morbidity and Mortality Weekly Report from February to June 2020 only included one county in California, which may not be representative of other counties within the state [8]. To estimate the actual number of infected persons in our community, we conducted a cross-sectional study during the height of the pandemic to estimate seroprevalence of SARS-CoV-2 infection.

\section{Methods}

The cross-sectional study was performed at a medium-sized nonacademic community hospital, St. Jude Medical Center, Fullerton, CA. We performed the immunoglobulin $\mathrm{G}(\mathrm{IgG})$, antibody testing for SARS-CoV-2 during the time period of July 15 , 2020 , to July 27,2020 . Testing was done on serum samples from patients who had visited an affiliated outpatient clinic or our emergency department. Serum samples are routinely stored for several days after being used for their intended laboratory purpose. These residual samples were used in our study to test for SARS-CoV-2 IgG antibodies prior to them being destroyed. Patients who visited multiple outpatient clinics and the emergency department during this time period were only counted once with the most recent positive result carried forward into analysis. The diagnostic platform utilized in the study was the Abbott Architect, which provided anti-SARS-CoV-2 IgG results. In addition to the anti-SARS-CoV-2 IgG test results, we performed a retrospective chart review and collected age, gender, ethnicity, race, and location of testing. The estimate of this study was the prevalence of seropositivity of anti-SARS-CoV-2 IgG and its $95 \%$ confidence interval. General population and study population data were obtained through geographic information systems data supplier, Esri. The institutional review board at Providence/St. Joseph Health Systems approved this study.

\section{Statistical Analysis}

Descriptive statistics were used to summarize the patient characteristics. Discrete data were presented as frequencies and percentages. The Pearson's chi-square or Fisher's test was used to measure the association between dichotomous variables. All tests were two-tailed, and a $p$ value of $<0.05$ was considered significant. The prevalence of COVID-19 in the population was measured as crude rates of positive tests without adjustments and weighted prevalence using the Orange County population in 2019. The percentages of IgG-positive patients were presented with $95 \%$ confidence intervals (CI).
Statistical analyses were carried out with SPSS version 26.0 (Chicago, IL, USA) and GraphPad Prism version 6.0 (San Diego, CA, USA).

\section{Results}

Nine hundred ninety-two antibody tests were performed. There were 127 test results that were unavailable due to missing patient data $(n=59)$ or were repeat patients $(n=68)$. The outpatient clinics cohort accounted for $56 \%(n=484)$ of the test results, with the remaining tests in the emergency department $(\mathrm{ED}), 44 \%(n=381)$. The positive percentage of SARSCoV-2 test was $9.4 \%$ (95\% CI: $0.08-0.12$ ). The positivity rates of the outpatient and ED setting differed, with an ER positivity rate of $14.2 \%$ (95\% CI: $0.11-0.18$ ) and outpatient positivity rate of $5.6 \%$ (95\% CI: $0.04-0.08$ ). Distribution of testing based on race and ethnicity are listed in Table 1 . The prevalence of a positive COVID-19 test was greatest in those that identified as Hispanic or Latino at $18.1 \%$. The rates of positivity in non-Hispanic or Latino and in patients who declined to state race or data not available were $6.7 \%$ in each group. The rates of positivity of the Hispanic/Latino population compared to the non-Hispanic/Latino population were significantly higher, with a relative risk of 2.73 (95\% CI: $1.8-4.1), p<0.0001$. Overall, the prevalence of positive antiSARS-CoV-2 IgG results was higher in the Hispanic/Latino population than those identified as non-Hispanic/Latino and individuals who declined to state, $18.1 \%$ vs $13.4 \%$, respectively. The distribution of the COVID-19-positive tests based on age is listed on Table 2. The group with the highest prevalence of positive tests was the 20-29-year-old group at $21.8 \%$ followed by the $40-49$-year-old group with $21.7 \%$. The rates of positive COVID-19 tests were more prevalent in patients under the age of 60 years $(15 \%)$ compared to those over 60 years $(5.4 \%), p<0.0001$, who are greater risk of severe illness. The age-adjusted prevalence of positive SARSCoV-2 IgG test was 5.98 cases per 100,000 based on the 2019 Orange County population.

\section{Discussion}

The global pandemic has highlighted the increasing need for accessible testing to limit the spread of SARS-CoV-2. Unidentified SARS-CoV-2-positive patients pose a risk of continued viral exposure and perpetuation of the pandemic. It has been estimated that as much as $86 \%$ of infections were undocumented with mild, limited, or asymptomatic [11, 12]. Our cross-sectional study evaluated the current positivity rate of COVID-19 in the community of northern Orange County and contributes to the underlying need for additional testing. The current level of seropositivity in the community indicates 


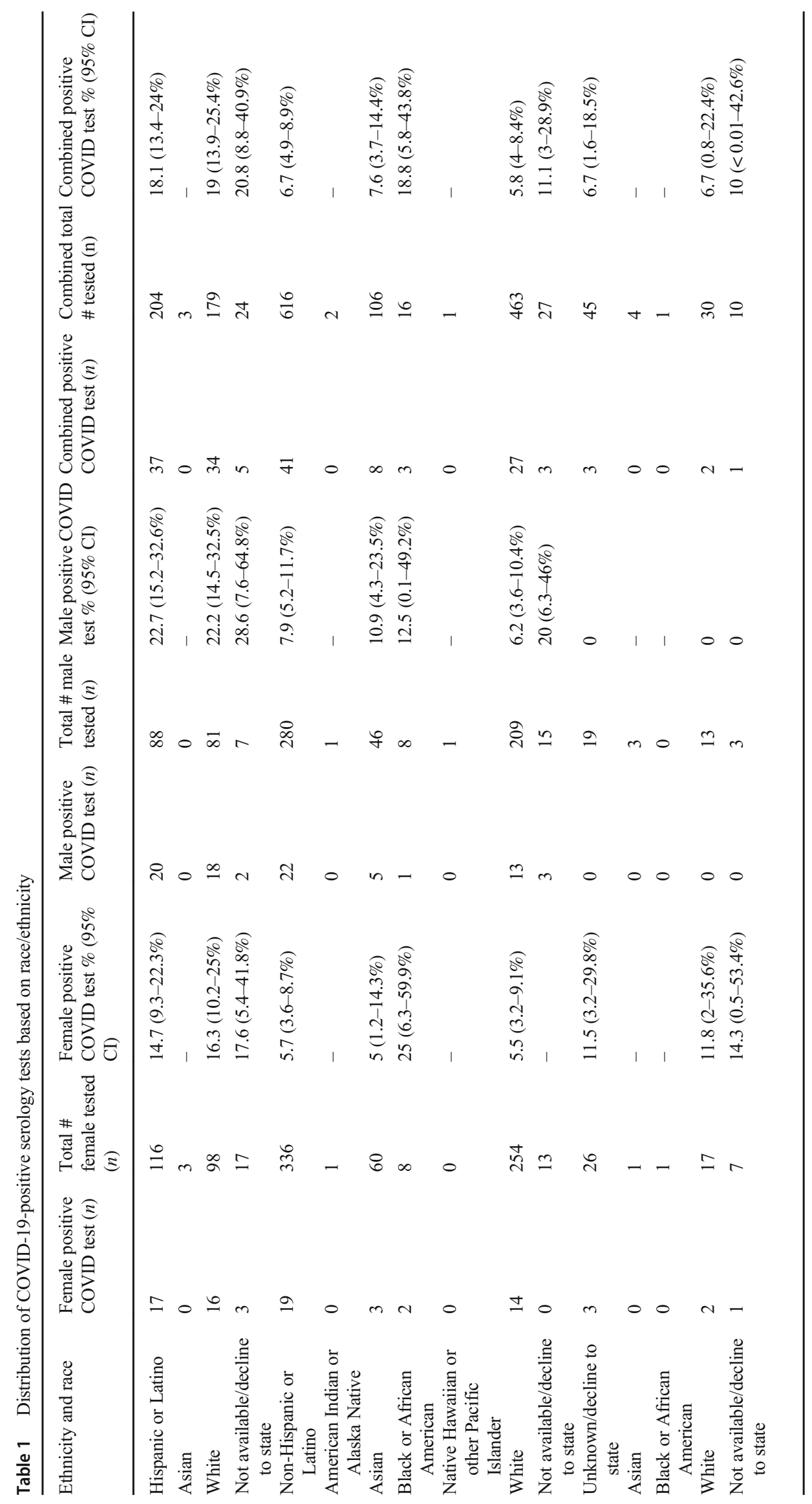




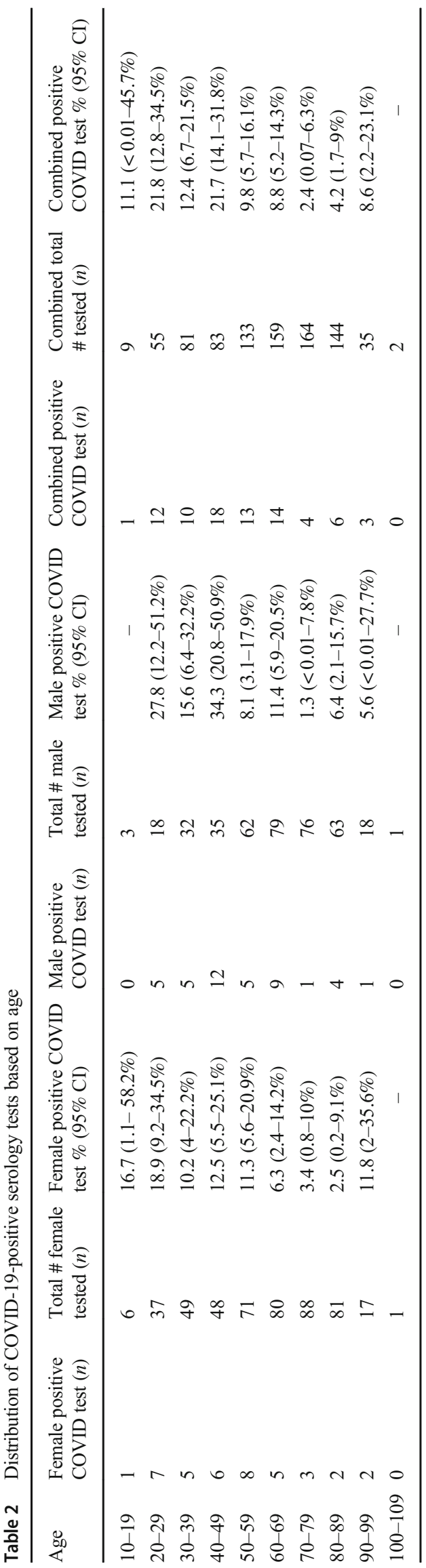

that more testing is required to identify a broader proportion of patients who have not sought medical care or had mild/ asymptomatic cases to determine if herd immunity is a possibility. Historically, immunity in $80 \%$ of the population is needed to provide a herd immunity effect [13]. An issue that arises with herd immunity is that distribution of immunity in the population may not be uniform. It is also still unknown how long immunity to COVID-19 will last after infection or vaccination. Despite this unknown, having a vaccine and vaccinating the population would be a prudent strategy to limit the spread of COVID-19 and reduce the associated morbidity and mortality.

From our cohort of patients, being of Hispanic/Latino origin had almost a threefold relative risk for COVID-19 than non-Hispanic/Latino White patients. The rates of positivity were disproportionately high in comparison to the demographics of the service area. The population of St. Jude Medical Center service area population consists of $45.8 \%$ that was identified as Hispanic/Latino in origin. These study results are consistent with the Morbidity and Mortality Weekly Report compiled by the CDC where the Hispanic/ Latino population had the highest rates of COVID-19 associated with workplace outbreaks [14]. According to the US Census Bureau, the population in California consists of 39.4\% Hispanic/Latino, but the percent of COVID-19 cases throughout California has disproportionately affected this group exceeding $60 \%$, which is also consistent with our study results $[15,16]$. In a Kaiser Family Foundation survey regarding knowledge, attitude, and good practices to reduce COVID-19 risk, racial/ethnic minorities were more likely to report engaging in better practices to reduce their risk of COVID-19 but had lower knowledge regarding the disease [17]. And yet minorities are more likely to acquire the infection and risk of mortality [8]. This could potentially be a health literacy issue or possibly due to the rates at which minorities work in essential worker positions that puts them at risk for COVID-19. Tools to improve health literacy regarding how to prevent COVID-19 acquisition needs to improve in this population. Strategies for this patient population should include targeted education and strong emphasis within this group for future immunization with a COVID-19 vaccine.

This study has several limitations. The study was retrospective and cross-sectional in design and reflects the internal flaws associated. Additionally, it is descriptive and only able to determine COVID-19 seroprevalence and not the true incidence in the population. It was conducted at the height of the pandemic in California, after the first wave and during the second wave of increased COVID-19 infections in California. We did not exclude patients visiting the emergency department for COVID-19 symptoms/diagnosis. We concede that the emergency department would increase the chances of having COVID-19, although the residual blood 
samples tested had no known connection to COVID-19 at the time of testing. The study population and results may not be generalizable to other institutions or counties, and selection bias is unavoidable. It should be noted that the study sample size was not large enough to precisely measure other racial/ ethnic populations as the number of patients from nonHispanic and non-White and patients in the age groups of 10-19 and >90 years of age were low and underrepresented. Thus, we were unable to estimate seroprevalence in these groups. The prevalence of COVID-19 is only increasing which will likely change the age-related positivity incidence. Our institution used the Abbott Architect testing system, which is approved under the FDA Emergency Use Authorization (EUA) with limited clinical studies using this device in real world settings; however, it has been found that the sensitivity and specificity at $\geq 14$ days from onset of symptoms is $100 \%$, and at $\geq 7$ days from onset, the sensitivity is $88.0 \%$ and specificity $99.6 \%$ [18].

In conclusion, the low antibody positivity rate and risk of morbidity and mortality associated with SARS-CoV-2 indicate the need for a vaccine. The population at greatest risk in our community and likely California for COVID-19 is those that were identified as Hispanic/Latino. This patient population should be considered for increased education on preventing transmission and acquisition of SARS-CoV-2 as well as being considered as a priority for vaccination once a vaccine is available.

\section{Compliance with Ethical Standards}

Conflict of Interest The authors declare that they have no conflict of interest.

Ethics Approval This study was approved by the Providence St. Joseph Health System Investigational Review Board.

Consent to Participate Not applicable.

Consent for Publication Not applicable.

\section{References}

1. World Health Organization. Clinical management of COVID-19: Interm Guidance. World Health Organization. https://www.who. int/publications/i/item/clinical-management-of-covid-19. License: CC BY-NC-SA 3.0 IGO. 2020. Accessed 29 Aug 2020.

2. Zhu N, Zhang D, Wang W, et al. A novel coronavirus from patients with pneumonia in China, 2019. N Engl J Med. 2020;382(8):72733.

3. Dong E, Du H, Gardner L. An interactive web-based dashboard to track COVID-19 in real time. Lancet Infect Dis. 2020;20(5):533-4.
4. Dong E DH, Gardner L. COVID-19 Dashboard by the Center for Systems Science and Engineering (CSSE) at Johns Hopkins University (JHU). Available at: https://coronavirus.jhu.edu/map. html. Accessed August 31.

5. Paakkari L, Okan O. COVID-19: health literacy is an underestimated problem. Lancet Public Health. 2020;5(5):e249 e50.

6. Centers-for-Disease-Control-and-Prevention. Implementation of mitigation strategies for communities with local COVID-19 transmission. Available at: https://www.cdc.gov/coronavirus/2019ncov/community/community-mitigation.html. Accessed August 31.

7. Bureau USC. QuickFacts. Available at: https://www.census.gov/ quickfacts/fact/table/US/PST045219. Accessed August 31.

8. Moore JT, Ricaldi JN, Rose CE, et al. Disparities in incidence of COVID-19 among underrepresented racial/ethnic groups in counties identified as hotspots during June 5-18, 2020 - 22 states, February-June 2020. MMWR Morb Mortal Wkly Rep. 2020;69(33):1122-6.

9. van Holm EJ, Wyczalkowski CK, Dantzler PA. Neighborhood conditions and the initial outbreak of COVID-19: the case of Louisiana. J Public Health (Oxf). 2020:fdaa147. https://doi.org/ 10.1093/pubmed/fdaa147.

10. Mahajan UV, Larkins-Pettigrew M. Racial demographics and COVID-19 confirmed cases and deaths: a correlational analysis of 2886 US counties. J Public Health (Oxf). 2020;42(3):445-7.

11. Li R, Pei S, Chen B, et al. Substantial undocumented infection facilitates the rapid dissemination of novel coronavirus (SARSCoV-2). Science. 2020;368(6490):489-93.

12. Mizumoto K, Kagaya K, Zarebski A, Chowell G. Estimating the asymptomatic proportion of coronavirus disease 2019 (COVID-19) cases on board the Diamond Princess cruise ship, Yokohama, Japan, 2020. Euro Surveill. 2020;25(10):2000180. https://doi.org/ 10.2807/1560-7917.ES.2020.25.10.2000180.

13. Kim TH, Johnstone J, Loeb M. Vaccine herd effect. Scand J Infect Dis. 2011;43(9):683-9.

14. Dyal JW, Grant MP, Broadwater K, et al. COVID-19 among workers in meat and poultry processing facilities - 19 states, April 2020. MMWR Morb Mortal Wkly Rep. 2020;69(18).

15. United States Census Bureau. QuickFacts. Available at: https:// www.census.gov/quickfacts/fact/table/US/PST045219. Accessed August 31.

16. California Department of Public Health. COVID-19 race and ethnicity data. Available at: https://www.cdph.ca.gov/Programs/CID/ DCDC/Pages/COVID-19/Race-Ethnicity.aspx. Accessed August 31 .

17. Alobuia WM, Dalva-Baird NP, Forrester JD, Bendavid E, Bhattacharya J, Kebebew E. Racial disparities in knowledge, attitudes and practices related to COVID-19 in the USA. J Public Health (Oxf). 2020;42(3):470-8.

18. Bryan A, Pepper G, Wener MH, Fink SL, Morishima C, Chaudhary A, et al. Performance characteristics of the Abbott Architect SARSCoV-2 IgG assay and seroprevalence in Boise, Idaho. J Clin Microbiol. 2020;58(8)8):e00941-20. https://doi.org/10.1128/JCM. 00941-20

Publisher's Note Springer Nature remains neutral with regard to jurisdictional claims in published maps and institutional affiliations. 